\title{
The Factors Associated With the Successful Outcomes of Percutaneous Disc Decompression in Patients With Lumbar Herniated Nucleus Pulposus
}

\author{
Sang Heon Lee, MD, PhD, Yong Jin Jeong, MD, Nack Hwan Kim, MD, \\ Hyeun Jun Park, MD, Hyun-Joon Yoo, MD, Soo Yung Jo, MD
}

Department of Physical Medicine and Rehabilitation, Korea University College of Medicine, Seoul, Korea

Objective To determine clinical and radiological factors that predict the successful outcome of percutaneous disc decompression (PDD) in patients with lumbar herniated nucleus pulposus (HNP).

Methods We retrospectively reviewed the clinical and radiological features of patients who underwent lumbar PDD from April 2009 to March 2013. Sixty-nine patients with lumbar HNP were studied. Clinical outcome was assessed by the visual analogue scale (VAS) and the Oswestry Disability Index (ODI). Multivariate logistic regression analysis was performed to assess relationship among clinical and radiological factors and the successful outcome of the PDD.

Results The VAS and the ODI decreased significantly at 1 year follow-up ( $\mathrm{p}<0.01)$. One year after PDD, the reduction of the VAS $(\triangle$ VAS) was significantly greater in the patients with pain for $<6$ months $(\mathrm{p}=0.03)$ and subarticular HNP $(\mathrm{p}=0.015)$. The reduction of the ODI $(\triangle \mathrm{ODI})$ was significantly greater in the patients with high intensity zone $(\mathrm{p}=0.04)$. Multivariate logistic regression analysis revealed the following 5 factors that were associated with the successful outcome after PDD: pain duration for $<6$ months (odds ratio $[\mathrm{OR}]=14.036 ; \mathrm{p}=0.006$ ), positive straight leg raising test $(\mathrm{OR}=8.425, \mathrm{p}=0.014)$, the extruded HNP $(\mathrm{OR}=0.106, \mathrm{p}=0.04)$, the sequestrated HNP ( $\mathrm{OR}=0.037, \mathrm{p}=0.026)$, and the subarticular HNP (OR=10.876, $\mathrm{p}=0.012)$.

Conclusion PDD provided significant improvement of pain and disability of patients. The results of the analysis indicated that the duration of pain $<6$ months, positive straight leg raising test, the subarticular HNP, and the protruded HNP were predicting factors associated with the successful response of PDD in patients with lumbar HNP.

Keywords Intervertebral disc displacement, Radiculopathy, Decompression, Regression analysis, Treatment outcome

Received May 8, 2015; Accepted June 1, 2015

Corresponding author: Yong Jin Jeong

Department of Physical Medicine and Rehabilitation, Korea University Anam Hospital, 73 Inchon-ro, Seongbuk-gu, Seoul 02841, Korea

Tel: +82-2-920-6471, Fax: +82-2-929-9951, E-mail: side_effect@naver.com

@ This is an open-access article distributed under the terms of the Creative Commons Attribution Non-Commercial License (http://creativecommons. org/licenses/by-nc/4.0) which permits unrestricted noncommercial use, distribution, and reproduction in any medium, provided the original work is properly cited.

Copyright $\odot 2015$ by Korean Academy of Rehabilitation Medicine

\section{INTRODUCTION}

Lumbar radicular pain due to a herniated nucleus pulposus (HNP) causes significant morbidity [1]. Lumbar HNP compresses nerve roots and its mechanical and inflammatory mechanisms play a role in causing pain [2]. In patients with small contained herniations, outcomes after microdiscectomy are worse than sequestrated 
herniations [3]. There are variable treatment options including physiotherapy, medication and epidural steroid injection for symptom relief. If symptoms persist after sufficient conservative treatment, more invasive methods are required. The disadvantage of surgery is potential damage to posterior elements of lumbar vertebra [4]. The advantage of percutaneous procedure is the potential for decreased surgical trauma, quick recovery time, and the low incidence of complications. Therefore, minimally invasive disc decompression procedures for treating a herniated disc have been developed. Percutaneous disc decompression (PDD) methods rely on the principle that small amounts removed from the disc will result in significant pain relief by lowering the intradiscal pressure [5]. Therefore, pressure on the nerve will be removed and radicular leg pain will be relieved. Clinical significant pain reduction and decreased disability for patients and safety of PDD is proved [6-8] and it is frequently performed for treating radicular pain caused by lumbar HNP.

In order to treat lumbar radicular pain more efficiently, appropriate subject selection for PDD is important. This study aimed to investigate the correlation among clinical and radiological factors and the outcome of PDD in the patients with lumbar HNP.

\section{MATERIALS AND METHODS}

\section{Subjects}

The study was approved by the Korea University Institutional Review Board in 2015. We retrospectively collected and analyzed data from April 2009 to March 2013. Patients who underwent PDD by L'DISQ (U\&I Co., Uijeong$\mathrm{bu}$, Korea) were analyzed. The inclusion criteria of the study were as follows: radicular symptoms and signs with for $\geq 1$ month despite conservative management (e.g., physiotherapy, selective nerve root blocks, and medication), and single-level lumbar HNP with neural contact as seen on lumbosacral spine magnetic resonance imaging (MRI) or MRI evidence of disc herniation with EMG evidence of lumbar radiculopathy. Patients with a progressive neurologic deficit including a cauda equine syndrome, previous lumbar disc operation, history of psychological disorder and radiological evidence of other pathologies that explain the clinical presentation (e.g., spinal stenosis and spinal cord tumor) were excluded.

\section{Review of the radiographic findings and clinical data}

We investigated the patients' medical records and MRI. We recorded the clinical characteristics and physical examination of the patients such as sex, age, smoking, duration of pain, and the result of the straight leg raising (SLR) test. The duration of pain that was longer than 6 months was defined as chronic pain. The straight leg raising test was considered positive when pain occurred before $60^{\circ}$ passive elevation from horizontal [9].

One independent physician analyzed the MRI without previous knowledge of the patients' symptoms. The MRI images were classified by HNP level, HNP type, HNP zone, nerve root compromise grade, disc degeneration grade, and the high intensity zone (HIZ). Classification of HNP type, HNP zone followed the standards used by the American Society of Spine Radiology, the American Society of Neuroradiology, and the North American Spine Society [10]. HNP type was divided into 'protrusion,' 'extrusion', and 'sequestration. HNP zone consisted of 'central, 'subarticular', 'foraminal', and 'extraforaminal'. Nerve root compromise grade was classified as grade 0 (normal), grade 1 (contact), grade 2 (deviation), and grade 3 (compression) [11]. Disc degenerative grade was assessed by 5 -scale degenerative grade by Pfirrmann et al. [12]. Grade 4 and 5 degeneration were defined as severe degeneration. The HIZ was defined as the local area with a high intensity signal in the posterior annulus fibrosus in the sagittal T2-weighted image [13].

\section{Procedure protocol}

PDD was performed on an outpatient basis. Preoperatively, prophylactic intravenous antibiotic was administered 30 minutes before the procedure. We monitored patients with electrocardiogram, pulse oximetry, and automated blood pressures. Sedative (20 $\mathrm{mg}$ of profopol) was administered as necessary during anesthetization of the skin and subcutaneous fascia.

The patients were placed in a prone position with the back mildly flexed on a surgical table. Fluoroscopic examination of the lumbar spine was performed to confirm intervertebral disc level and determine the appropriate level of needle [6]. We used a standard posterior lateral approach to the intervertebral disc, as previously described [14]; however, we modified the technique to approach the intervertebral disc more laterally so that the introducer needle would contact the disc margin rather 
than the midline.

A 25-gauge needle was first inserted into the ipsilateral side of target disc nucleus and 1 to $2 \mathrm{~mL}$ of contrast was injected to outline the disc herniation. Then, $\mathrm{C}$-arm was rotated to position the lateral margin of the contralateral superior articular process as visualized in the oblique position. This typically required rotating the $\mathrm{C}$-arm $15^{\circ}$ from a zero-degree lateral projection. The skin and subcutaneous tissue were infiltrated with $1 \%$ lidocaine. We manually curved the 16-gauge introducer needle approximately $15^{\circ}$ in the distal, $1 \mathrm{~cm}$ from the distal tip. The introducer needle was advanced toward the lateral edge of the superior articular process. Once the lateral edge was touched, we rotated the needle to point toward the midline. As the needle tip was directed toward the midline, the anteroposterior (AP) projection was checked intermittently to assure that the needle tip was always lateral of the medial border of the pedicle. The patient was asked to report any buttock or leg pain. The advancement of the needle was controlled by rotating the direction of the bent needle tip. Entering the herniation was identified by a sudden loss of resistance. After confirming the introducer needle position with the lateral and AP view, the wand was advanced to the center of the herniated disc under fluoroscopic monitoring (Figs. 1, 2). Before ablation, motor nerve stimulation was performed to confirm that the needle was not close to the nerve root. During the ablation, the tip of the wand was continuously rotated and moved back and forth to increase the ablated volume of the disc [6]. The entire procedure was monitored and evaluated by the C-arm fluoroscopy.

\section{Outcome measures}

The effectiveness of PDD was investigated using the vi-
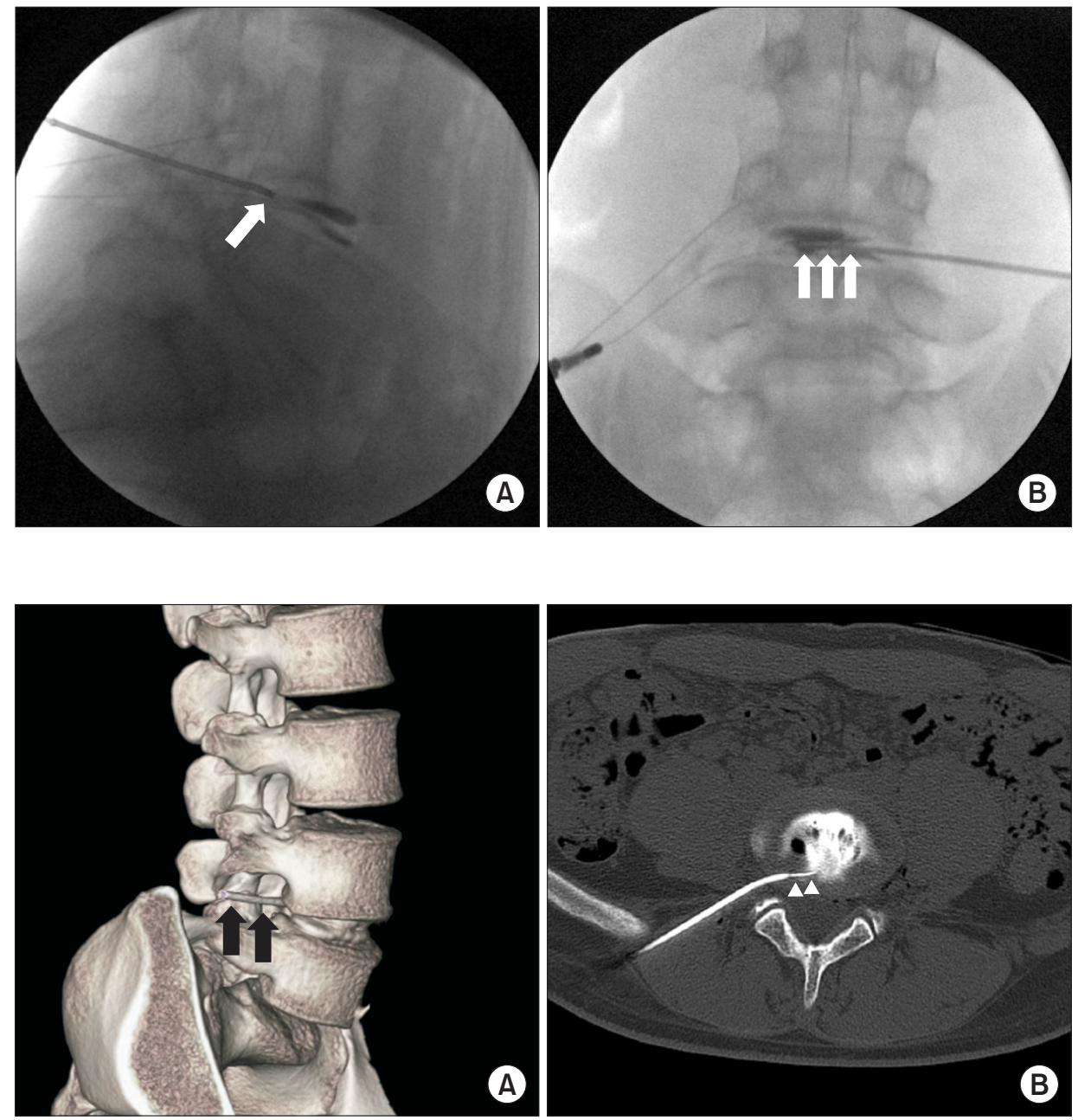

Fig. 1. Percutaneous disc decompression was performed using L'DISQ catheter with fluoroscopic guidance. After the injection of contrast media, the C-arm fluoroscopic view shows the tip of wand (arrows) within a posterior lumbar intervertebral disc. (A) Lateral view, (B) anteroposteior view.

Fig. 2. A computed tomographic reconstruction image of the L'DISQ wand after percutaneous disc decompression (PDD) is shown. (A) The introducer needle (black arrows) was inserted posterior to the annulus fibrosus. (B) From the axial view of computed tomographic image, the tip of the PDD wand (white arrowheads) is seen within the intervertebral disc. 
sual analogue scale (VAS) and the Oswestry Disability Index (ODI). Baseline data was obtained before the procedure. After the procedure, patients visited our outpatient department and answered the VAS and the ODI after 1-, 3-, 6-, and 12-month. We defined the successful outcome as $>50 \%$ reduction of the VAS.

\section{Statistical analysis}

The improvement of pain and disability after percutaneous disc decompression were analyzed by the Wilcoxon signed-rank test. The Mann-Whitney U test, the Kruskal-Wallis test were used for analysis of difference between the clinical outcome and the presence of the patients' clinical and radiological data such as sex, smoking, duration of pain, SLR test, HNP level, HNP type, HNP zone, nerve root compromise grade, disc degeneration grade and HIZ. The Spearman correlation analysis was used to measure the correlation between age and the clinical outcomes. Univariate logistic regression analysis was performed on each variables to determine the crude odds ratios of successful outcome. After that, multivariate analysis was performed by logistic regression using the above-mentioned independent variables, and the successful outcome of PDD as the dependent variable. Sta- tistical analysis were performed using SPSS Statistics ver. 20 software (IBM, Armonk, NY, USA). Significance was determined when the $\mathrm{p}$-value was $<0.05$.

\section{RESULTS}

\section{Sample characteristics}

PDDs were performed in 109 patients from April 2009 to March 2013. Follow-up data were obtained in 83 patients. Seven patients were excluded due to prior lumbar surgery and 7 patients were excluded because of multi-level lumbar HNP. Sixty-nine patients, with mean age of 42.71 years (standard deviation of 12.76 years) were included in this study where $43(62.3 \%)$ were male. Mean duration of symptoms was 19.90 months, ranging from 1 to 124 months. The basic clinical characteristics are shown in Table 1. There were no significant differences of baseline VAS and ODI according to subgroup analysis (Table 1).

\section{Clinical outcome after PDD}

Clinical outcome was investigated through the medical records. The VAS score decreased significantly from $6.59 \pm 2.00$ to $2.46 \pm 2.00$ at 1 year evaluation $(\mathrm{p}<0.01)$ (Fig. 3). The ODI score significantly decreased from

Table 1. Baseline characteristics of the study subjects

\begin{tabular}{|c|c|c|c|c|c|}
\hline & Value & Baseline VAS & p-value ${ }^{\text {a) }}$ & Baseline ODI & p-value ${ }^{a)}$ \\
\hline Sex & & & 0.121 & & 0.069 \\
\hline Male & $43(62.3)$ & $6.88 \pm 1.98$ & & $43.93 \pm 16.92$ & \\
\hline Female & $26(37.7)$ & $6.12 \pm 1.99$ & & $51.02 \pm 15.96$ & \\
\hline Age (yr) & $42.71 \pm 12.76(19-71)$ & & 0.776 & & 0.990 \\
\hline $18-40$ & $29(42.0)$ & $6.83 \pm 1.63$ & & $46.33 \pm 19.55$ & \\
\hline$>40$ & $40(58.0)$ & $6.42 \pm 2.24$ & & $46.79 \pm 14.76$ & \\
\hline Smoking & & & 0.324 & & 0.402 \\
\hline Yes & $15(21.7)$ & $7.13 \pm 1.96$ & & $43.93 \pm 19.53$ & \\
\hline No & $54(78.3)$ & $6.44 \pm 2.01$ & & $47.34 \pm 16.09$ & \\
\hline Duration of pain (mo) & $19.90 \pm 26.63(1-124)$ & & 0.575 & & 0.238 \\
\hline $1-6$ & $28(40.6)$ & $6.82 \pm 1.70$ & & $49.51 \pm 19.69$ & \\
\hline$>6$ & $41(59.4)$ & $6.44 \pm 2.19$ & & $44.62 \pm 14.44$ & \\
\hline HNP level & & & 0.201 & & 0.201 \\
\hline $\mathrm{L} 3 / 4$ & $5(7.2)$ & $6.60 \pm 1.95$ & & $33.96 \pm 14.71$ & \\
\hline $\mathrm{L} 4 / 5$ & $37(56.6)$ & $6.86 \pm 2.10$ & & $48.83 \pm 17.00$ & \\
\hline $\mathrm{L} 5 / \mathrm{S} 1$ & 27 (39.1) & $6.22 \pm 1.89$ & & $45.88 \pm 16.33$ & \\
\hline
\end{tabular}

Values are presented as number (\%) or mean \pm standard deviation (range).

VAS, visual analogue scale; ODI, Oswestry Disability Index; HNP, herniated nucleus pulposus.

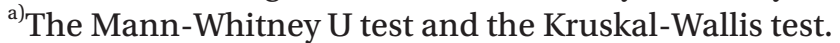


$46.60 \pm 16.80$ to $25.02 \pm 16.68$ at 1 year follow-up $(\mathrm{p}<0.01)$ (Fig. 4). Eight patients who had prolonged pain underwent conventional microdiscectomy after PDD. There were no major complications including nerve damage, epidural hematoma or spondylodiscitis.

The improvement of pain ( $\triangle V A S)$ after PDD according to the clinical, radiological factors

The change of VAS according to the factors were presented in Table 2 . The improvement of pain ( $\triangle$ VAS) between the baseline and 1 year follow-up was calculated. There were no significant differences in baseline VAS among subgroups. One year after the PDD, $\triangle$ VAS of patients with their pain $<6$ months was significantly greater than that with chronic pain patients $(\mathrm{p}=0.03) . \Delta$ VAS was significantly different between the 3 groups by HNP zone $(\mathrm{p}=0.04)$. Post hoc comparison showed that $\triangle$ VAS of the subarticular HNP was considerably better than that of the central HNP ( $p=0.015)$. We were unable to compare the effectiveness in the extraforaminal zone since there were no patients enrolled. Difference of $\triangle$ VAS by SLR test showed borderline significance between the 2 groups $(p=0.06)$. The following factors showed no significant differences with the $\triangle$ VAS: sex, smoking, HNP level, HNP type, nerve root compromise grade, disc degeneration grade, and HIZ. Age was not significantly correlated with $\triangle$ VAS by the Spearman correlation analysis $(r=0.012$, $\mathrm{p}=0.93)$.

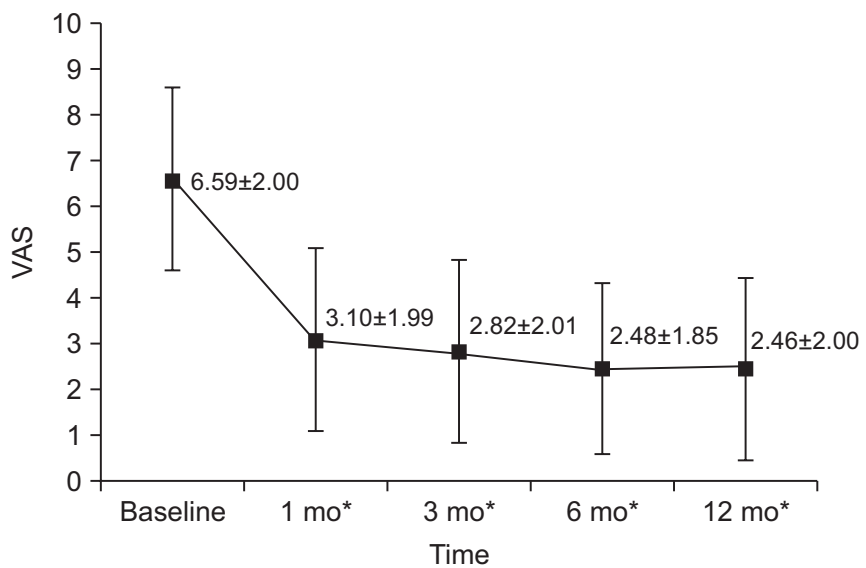

Fig. 3. Changes of the visual analogue scale (VAS) after percutaneous disc decompression at 1-, 3-, 6- and 12-month of follow-up assessment. Values are presented as mean \pm standard deviation. * Significant differences $(\mathrm{p}<0.01)$ from the baseline.
The improvement of disability ( $\triangle O D I)$ after PDD according to the clinical, radiological factors

The improvement of disability ( $\Delta \mathrm{ODI})$ between baseline and 1 year follow-up was calculated. The statistical differences of $\Delta \mathrm{ODI}$ among the various factors were analyzed. There were no significant differences in baseline ODI among subgroups. ODI of the patients with HIZ was significantly better than that of the patients without HIZ ( $p=0.04$ ). Difference of $\Delta$ ODI by duration of pain showed borderline significance between the 2 groups $(\mathrm{p}=0.05)$. $\triangle$ ODI was not significantly different among sex, smoking, SLR test, HNP level, HNP type, HNP zone, nerve root compromise grade, and disc degeneration grade (Table 2). Age was not significantly correlated with $\Delta$ ODI by the Spearman correlation analysis $(\mathrm{r}=-0.156, \mathrm{p}=0.23)$.

Associations with patient's clinical and radiological characteristics and the successful outcome of PDD

The associations between the successful outcome of PDD and patients' clinical and radiological characteristics were summarized in Table 3. Univariate analysis indicated significant differences for pain duration, SLR test, and HNP zone. Nerve root compromise grade was not calculated for the analysis because of small sample size in the deviation grade. When a dependent variable was set as a binary outcome by the presence of successful outcome after PDD, multivariate binary logistic regression analysis identified 5 independent predictors: pain duration of $<6$ months (odds ratio [OR], 14.036; 95\%

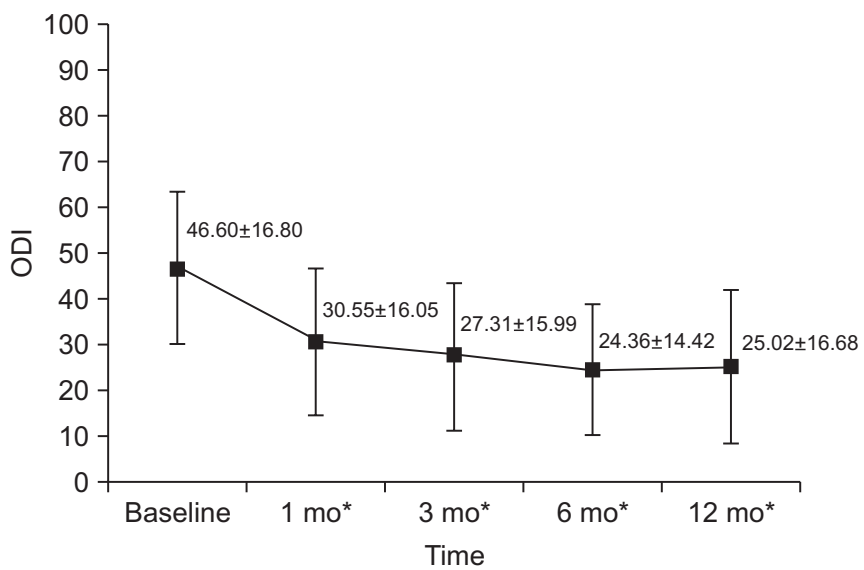

Fig. 4. Changes of the Oswestry Disability Index (ODI) after the percutaneous disc decompression at 1-, 3-, 6- and 12 -month of follow-up assessment. *Significant differences $(\mathrm{p}<0.01)$ from the baseline. 
Table 2. The reduction of VAS ( $\triangle$ VAS) and ODI ( $\triangle$ ODI) between baseline and 1 year follow-up after PDD according to clinical and radiological variables

\begin{tabular}{|c|c|c|c|c|c|}
\hline & No. (\%) & $\Delta$ VAS & p-value ${ }^{\text {a) }}$ & $\Delta$ ODI & p-value ${ }^{\text {a) }}$ \\
\hline Sex & & & 0.44 & & 0.72 \\
\hline Male & $43(62.3)$ & $4.35 \pm 2.96$ & & $23.71 \pm 20.87$ & \\
\hline Female & $26(37.7)$ & $3.69 \pm 3.17$ & & $23.71 \pm 18.26$ & \\
\hline Smoking & & & 0.09 & & 0.29 \\
\hline Yes & $15(21.7)$ & $5.07 \pm 3.22$ & & $26.49 \pm 16.41$ & \\
\hline No & $54(78.3)$ & $3.83 \pm 2.96$ & & $20.22 \pm 19.10$ & \\
\hline Duration of pain (mo) & & & 0.03 & & 0.05 \\
\hline $1-6$ & $28(40.6)$ & $5.11 \pm 2.25$ & & $26.65 \pm 24.68$ & \\
\hline$>6$ & $41(59.4)$ & $3.41 \pm 3.32$ & & $18.11 \pm 17.80$ & \\
\hline SLR test & & & 0.06 & & 0.38 \\
\hline Positive & $42(60.9)$ & $5.07 \pm 2.24$ & & $23.81 \pm 16.56$ & \\
\hline Negative & $27(39.1)$ & $3.48 \pm 3.33$ & & $20.15 \pm 20.80$ & \\
\hline HNP level & & & 0.45 & & 0.85 \\
\hline $\mathrm{L} 3 / 4$ & $5(7.2)$ & $4.40 \pm 3.36$ & & $17.96 \pm 8.38$ & \\
\hline $\mathrm{L} 4 / 5$ & $37(53.6)$ & $4.35 \pm 3.42$ & & $22.40 \pm 20.78$ & \\
\hline $\mathrm{L} 5 / \mathrm{S} 1$ & $27(39.1)$ & $3.70 \pm 2.41$ & & $21.13 \pm 18.76$ & \\
\hline HNP type & & & 0.80 & & 0.87 \\
\hline Protrusion & $23(33.3)$ & $4.04 \pm 3.03$ & & $19.02 \pm 18.28$ & \\
\hline Extrusion & $35(50.7)$ & $4.37 \pm 2.88$ & & $22.94 \pm 19.17$ & \\
\hline Sequestration & $11(15.9)$ & $3.36 \pm 3.64$ & & $22.58 \pm 22.38$ & \\
\hline HNP zone & & & 0.04 & & 0.83 \\
\hline Central & $40(58.0)$ & $3.42 \pm 2.79^{b)}$ & & $22.91 \pm 18.95$ & \\
\hline Subarticular & $25(36.2)$ & $5.04 \pm 3.24^{\mathrm{b})}$ & & $19.53 \pm 20.91$ & \\
\hline Foraminal & $4(5.8)$ & $5.00 \pm 2.94$ & & $21.08 \pm 11.54$ & \\
\hline Extraforaminal & $0(0.0)$ & - & & - & \\
\hline Neural compromise grade & & & 0.14 & & 0.86 \\
\hline Normal & $12(17.4)$ & $2.83 \pm 3.12$ & & $20.08 \pm 19.64$ & \\
\hline Contact & $20(29.0)$ & $4.25 \pm 3.09$ & & $23.49 \pm 21.47$ & \\
\hline Deviation & $4(5.8)$ & $6.75 \pm 0.95$ & & $29.35 \pm 23.76$ & \\
\hline Compression & $33(47.8)$ & $4.15 \pm 2.99$ & & $20.03 \pm 17.64$ & \\
\hline Disc degeneration grade & & & 0.82 & & 0.17 \\
\hline 1 & $0(0.0)$ & - & & - & \\
\hline 2 & $9(13.0)$ & $4.44 \pm 3.50$ & & $29.35 \pm 23.46$ & \\
\hline 3 & $8(11.6)$ & $4.88 \pm 2.53$ & & $15.03 \pm 11.89$ & \\
\hline 4 & $34(49.3)$ & $3.94 \pm 3.33$ & & $19.94 \pm 14.93$ & \\
\hline 5 & $18(26.1)$ & $3.89 \pm 2.54$ & & $27.47 \pm 24.70$ & \\
\hline HIZ & & & 0.15 & & 0.04 \\
\hline Present & $8(11.6)$ & $5.63 \pm 1.92$ & & $35.14 \pm 17.81$ & \\
\hline Absent & $61(88.4)$ & $3.90 \pm 3.11$ & & $19.80 \pm 18.80$ & \\
\hline
\end{tabular}

Values are presented as number (\%) or mean \pm standard deviation.

VAS, visual analogue scale; ODI, Oswestry Disability Index; HNP, herniated nucleus pulposus; SLR, straight leg raising; HIZ, high intensity zone; PDD, percutaneous disc decompression.

$\triangle$ VAS $=$ VAS 1 year after PDD - baseline VAS

$\triangle$ ODI $=$ ODI 1 year after PDD - baseline ODI

a) The Mann-Whitney U test and the Kruskal-Wallis test.

${ }^{b}$ Significant difference between two groups by the Mann-Whitney U test $(p=0.015)$. 
Table 3. Unadjusted and adjusted OR of the successful response after PDD

\begin{tabular}{|c|c|c|c|c|}
\hline Variable & Unadjusted OR (95\% CI) & p-value ${ }^{\text {a) }}$ & Adjusted OR (95\% CI) & p-value ${ }^{\text {b) }}$ \\
\hline \multicolumn{5}{|l|}{ Sex } \\
\hline Male & 1.000 & & 1.000 & \\
\hline Female & $0.591(0.214-1.629)$ & 0.309 & $0.586(0.106-3.230)$ & 0.586 \\
\hline \multicolumn{5}{|l|}{ Age (yr) } \\
\hline Young (18-40) & 1.000 & & 1.000 & \\
\hline$>40$ & $0.750(0.272-2.068)$ & 0.578 & $0.971(0.201-4.682)$ & 0.971 \\
\hline \multicolumn{5}{|l|}{ Smoking } \\
\hline No & 1.000 & & 1.000 & \\
\hline Yes & $2.545(0.641-10.102)$ & 0.184 & $3.343(0.407-27.488)$ & 0.261 \\
\hline \multicolumn{5}{|l|}{ Pain duration (mo) } \\
\hline Chronic (>6) & 1.000 & & 1.00 & \\
\hline $1-6$ & $5.714(1.682-19.411)$ & 0.005 & $14.036(2.134-92.309)$ & 0.006 \\
\hline \multicolumn{5}{|l|}{ SLR test } \\
\hline Negative & 1.000 & & 1.000 & \\
\hline Positive & $3.635(1.156-11.427)$ & 0.027 & $8.425(1.548-45.852)$ & 0.014 \\
\hline \multicolumn{5}{|l|}{ HNP level } \\
\hline $\mathrm{L} 3 / 4$ & 1.000 & & 1.000 & \\
\hline $\mathrm{L} 4 / 5$ & $0.411(0.042-4.055)$ & 0.446 & $0.924(0.037-23.106)$ & 0.961 \\
\hline $\mathrm{L} 5 / \mathrm{S} 1$ & $0.500(0.049-5.154)$ & 0.560 & $0.930(0.036-24.053)$ & 0.965 \\
\hline \multicolumn{5}{|l|}{ HNP type } \\
\hline Protrusion & 1.000 & & 1.000 & \\
\hline Extrusion & $0.740(0.241-2.274)$ & 0.600 & $0.106(0.013-0.899)$ & 0.040 \\
\hline Sequestration & $0.766(0.168-3.486)$ & 0.730 & $0.037(0.002-0.674)$ & 0.026 \\
\hline \multicolumn{5}{|l|}{ HNP zone } \\
\hline Central & 1.000 & & 1.000 & \\
\hline Subarticular & $4.295(1.246-14.806)$ & 0.021 & $10.876(1.705-69.361)$ & 0.012 \\
\hline Foraminal & $0.818(0.105-6.397)$ & 0.848 & $0.586(0.016-21.902)$ & 0.773 \\
\hline Extraforaminal & - & 1.000 & - & 1.000 \\
\hline \multicolumn{5}{|l|}{ Disc degeneration grade } \\
\hline Non-severe (grade $1,2,3$ ) & 1.000 & & 1.000 & \\
\hline Severe (grade 4,5 ) & $0.550(0.156-1.940)$ & 0.353 & $1.133(0.148-8.701)$ & 0.904 \\
\hline \multicolumn{5}{|l|}{ HIZ } \\
\hline Absent & 1.000 & & 1.000 & \\
\hline Present & $4.237(0.489-36.677)$ & 0.190 & $14.588(0.769-276.674)$ & 0.074 \\
\hline
\end{tabular}

OR, odds ratio; CI, confidence interval; SLR, straight leg raising; HIZ, high intensity zone; PDD, percutaneous disc decompression.

${ }^{a)}$ Unadjusted OR by logistic regression analysis.

${ }^{b)}$ Adjusted OR by multivariate logistic regression analysis.

confidence interval [CI], 2.134-92.309), positive SLR test (OR, 8.425; 95\% CI, 1.548-45.852), the extruded HNP (OR, 0.106; 95\% CI, 0.013-0.899), the sequestrated HNP (OR, 0.037; 95\% CI, 0.002-0.674), and the subarticular
HNP (OR, 10.876; 95\% CI, 1.705-69.361). HIZ (OR, 14.588; 95\% CI, 0.769-276.674) showed borderline significance $(\mathrm{p}=0.074)$. No significant associations were observed for sex, young age ( $<40$ years), smoking, HNP level, and se- 
vere disc degeneration.

\section{DISCUSSION}

The factors that affect the success of PDD are still being studied [1]. To the best of our knowledge, this is the first study to analyze various clinical and radiological factors for predicting outcome after lumbar PDD. In this study, we evaluated the treatment outcomes of the PDD and investigated several clinical and radiological factors for predicting successful response of PDD. We found that VAS and ODI were significantly reduced after PDD for patients with lumbar HNP at 1 year follow-up. Pain improvement was better in patients with non-chronic pain and the subarticular HNP. Disability improvement was better in patients with HIZ. Patients with pain for $<6$ months, positive SLR test and the subarticular HNP were more likely to achieve the successful outcome after PDD. Patients with the extruded and sequestrated HNP were less likely to get the successful outcome after PDD.

An absolute indication for lumbar disc surgery is a progressive neurologic deficit including a cauda equine syndrome [15]. Except in that case, we should choose a proper management method to treat patients' pain and disability. A drawback of lumbar spine surgery is that it can damage posterior elements of lumbar vertebra by muscle dissection, bone removal or long surgical incisions [4]. PDD is performed with local anesthesia, so it is useful for those patients who cannot undergo conventional open discectomy under general anesthesia. Patients can also resume daily life activities more quickly.

Patients with lumbar HNP can undergo conservative treatment or epidural steroid injection to control symptoms. Conservative care provides resolution of symptoms, often coinciding with regression of HNP [16]. However, the natural history of HNP is often self-limited and pain gradually progresses over weeks or months. Erginousakis et al. [17] reported that PDD shows greater amelioration of symptoms at 12- and 24-month followup than conservative therapy. Another study showed that patients with a contained lumbar HNP who were treated with PDD had significantly reduced pain and disability than those treated using repeated transforaminal epidural steroid injection [7]. Therefore, better long-term outcome after PDD is expected. In the current study, the pain and disability were significantly reduced after PDD in lumbar HNP patients after 1 year follow-up. Thus, PDD can be recommended as an alternative treatment option after sufficient conservative treatment to treat radicular pain related to lumbar HNP in patients without progressive neurologic deficit.

Our study included patients with pain duration of $>1$ month. There are several studies to evaluate the efficacy of PDD that include patients with persistent symptoms for $>4$ weeks $[6,7,18]$. We aimed to verify the effectiveness of PDD as a treatment options in patients with acute lumbar HNP. There were 14 patients whose pain duration was $<3$ months. Eight patients had epidural steroid injections but injections were not helpful. Six patients had severe pain ( $>7$ VAS) and they were recommended surgery at other clinics. Because of severe pain, they could not tolerate conservative treatment and opted for non-surgical method. However, further study is needed to distinguish the effect of PDD and the natural history of lumbar disc herniation.

Karaman et al. [8] proved the effectiveness of PDD for chronic radicular pain. In our study, the patients with chronic radicular pain also showed significant pain and disability improvement 1 year after PDD $(\mathrm{p}<0.01)$. However, there was a significant difference of $\triangle$ VAS between chronic and non-chronic patients. One possible explanation is that chronic pain itself can modify sensory processing in the somatosensory system. Increased excitability, decreased inhibition and structural reorganization process in the spinal cord occur in chronic neuropathic disorders [19].

The SLR test as performed in clinical practice has a strong correlation with various parameters that signify the pain level of the patient [20]. A direct mechanical pressure by the lumbar HNP is the proposed explanation of positive SLR test. Positive SLR test was one of the factors associated with the successful outcome in our study. It can be explained by the effectiveness of PDD. By lowering intradiscal pressure and removing herniated disc, mechanical compression causing positive SLR test was thought to be successfully relieved after PDD.

From our study, the subarticular HNP showed better improvement of pain than the central HNP. Patients with the central HNP also showed significant pain and disability improvement 1 year after $\operatorname{PDD}(\mathrm{p}<0.01)$, but the $\triangle$ VAS were different between the central and subarticular HNP groups. Knop-Jergas et al. [21] reported a poorer clinical 
outcome of central lumbar HNP than other HNP after lumbar discectomy. The reason seems to be the difficulty of approaching the central disc with a peculiar derangement of disc architecture. Approaching central HNP during PDD, also needs more effort because of its deep location. If we use conventional PDD devices, the difference of $\triangle$ VAS between the subarticular HNP and central HNP could have been greater than the result of this study. In contrast to most PDD devices, navigable PDD device (L'DISQ) has a flexible navigable tip that can be directed to the desired position by rotating control wheel, and this direct approach to the herniated disc material allows effective disc decompression [6].

Bokov et al. [18] studied that the size of the disc protrusion does not significantly affect the outcome of PDD, but PDD performed in cases of uncontained disc herniation have a significantly higher rate of unsatisfactory results versus microdiscectomy. Our study likewise showed that the successful outcome was associated with the HNP types by multivariate logistic regression. Patients with the extruded or sequestrated HNP also showed significant pain $(\mathrm{p}<0.01$ and $\mathrm{p}=0.021$, respectively) and disability $(\mathrm{p}<0.01$ and $\mathrm{p}=0.026$, respectively) improvement 1 year after PDD, however, they were less likely to achieve the successful outcome after PDD than patients with the protruded HNP. This result supports the previous principal that PDD is more useful in the contained disc. The decrease of pressure inside extruded and sequestrated disc could be lower than in cases of protruded disc herniations. The amount of ablated disc material could be different, but further research is needed for this hypothesis.

Our study showed that patients with HIZ had more disability improvement than those without HIZ after PDD. Kim et al. [22] reported that the incidence of HIZ was significantly higher in patients with acute severe lower back pain than in patients in the control groups. Kang et al. [23] also showed HIZ has meaning when it accompanies disc protrusion. On the basis of these studies, we can assume that HIZ with disc protrusion is associated with acute inflammatory process causing pain. Therefore, prompt removal of pathologic disc material could contribute to a better clinical outcome in this study.

There were some limitations in our study that should be considered in the interpretation of results. First, this was a retrospective study with a small sample sized; the VAS and ODI of 26 patients could not be evaluated because of lack of medical records. Secondly, we did not analyze patients' socioeconomic state or other comorbidity such as diabetes mellitus. Additionally, we did not measure herniation size, since all the patients had HNP less than $1 / 3$ of spinal canal. Lastly, we used navigable PDD device, which may affect the clinical outcomes.

Our analysis identified the pain duration of $<6$ months, positive SLR test, the subarticular HNP, and the protruded HNP as predicting factors associated with the successful response of PDD in the patients with lumbar HNP. However, no significant associations were observed for sex, young age ( $<40$ years), smoking, HNP level, and severe disc degeneration. We also confirmed the effectiveness of PDD in lumbar HNP patients with radicular pain. Further prospective studies should be undertaken to define this correlation.

\section{CONFLICT OF INTEREST}

The first author invented the device that was used for the percutaneous disc decompression and he has an approximate $4.0 \%$ royalty interest in the device. The corresponding author has no conflict of interest.

\section{ACKNOWLEDGMENTS}

This research was supported by a grant of the Korea Health Technology R\&D Project through the Korea Health Industry Development Institute (KHIDI), funded by the Ministry of Health \& Welfare, Republic of Korea (No. HI14C3477).

\section{REFERENCES}

1. Ong D, Chua NH, Vissers K. Percutaneous disc decompression for lumbar radicular pain: a review article. Pain Pract 2014 Oct 29 [Epub]. http://dx.doi. org/10.1111/papr.12250.

2. Kuslich SD, Ulstrom CL, Michael CJ. The tissue origin of low back pain and sciatica: a report of pain response to tissue stimulation during operations on the lumbar spine using local anesthesia. Orthop Clin North Am 1991;22:181-7.

3. Dewing CB, Provencher MT, Riffenburgh RH, Kerr S, Manos RE. The outcomes of lumbar microdiscectomy in a young, active population: correlation by hernia- 
tion type and level. Spine (Phila Pa 1976) 2008;33:338.

4. Brouwer PA, Brand R, van den Akker-van Marle ME, Jacobs WC, Schenk B, van den Berg-Huijsmans AA, et al. Percutaneous laser disc decompression versus conventional microdiscectomy in sciatica: a randomized controlled trial. Spine J 2015; 15:857-65.

5. Singh V, Piryani C, Liao K, Nieschulz S. Percutaneous disc decompression using coblation (nucleoplasty) in the treatment of chronic discogenic pain. Pain Physician 2002;5:250-9.

6. Lee SH, Derby R, Sul DG, Hong JW, Kim GH, Kang S, et al. Efficacy of a new navigable percutaneous disc decompression device (L'DISQ) in patients with herniated nucleus pulposus related to radicular pain. Pain Med 2011;12:370-6.

7. Gerszten PC, Smuck M, Rathmell JP, Simopoulos TT, Bhagia SM, Mocek CK, et al. Plasma disc decompression compared with fluoroscopy-guided transforaminal epidural steroid injections for symptomatic contained lumbar disc herniation: a prospective, randomized, controlled trial. J Neurosurg Spine 2010;12: 357-71.

8. Karaman H, Tufek A, Olmez Kavak G, Yildirim ZB, Temel V, Celik F, et al. Effectiveness of nucleoplasty applied for chronic radicular pain. Med Sci Monit 2011;17:CR461-466.

9. Charnley J. Orthopaedic signs in the diagnosis of disc protrusion: with special reference to the straight-legraising test. Lancet 1951;1:186-92.

10. Fardon DF, Milette PC; Combined Task Forces of the North American Spine Society, American Society of Spine Radiology, and American Society of Neuroradiology. Nomenclature and classification of lumbar disc pathology. Recommendations of the Combined task Forces of the North American Spine Society, American Society of Spine Radiology, and American Society of Neuroradiology. Spine (Phila Pa 1976) 2001;26:E93E113.

11. Pfirrmann CW, Dora C, Schmid MR, Zanetti M, Hodler J, Boos N. MR image-based grading of lumbar nerve root compromise due to disk herniation: reliability study with surgical correlation. Radiology 2004; 230:583-8.
12. Pfirrmann CW, Metzdorf A, Zanetti M, Hodler J, Boos N. Magnetic resonance classification of lumbar intervertebral disc degeneration. Spine (Phila Pa 1976) 2001;26:1873-8.

13. Aprill C, Bogduk N. High-intensity zone: a diagnostic sign of painful lumbar disc on magnetic resonance imaging. Br J Radiol 1992;65:361-9.

14. Derby R, Lee SH, Kim BJ. Discography. In: Slipman CS, editor. Interventional spine: an algorithmic approach. Philadelphia: Saunders; 2008. p. 291-302.

15. Herkowitz HN, Garfin SR, Eismont FJ, Bell GR, Balderston RA. Rothman-Simeone the spine. 6th ed. Philadelphia: Elsevier Health Sciences; 2011.

16. Splendiani A, Puglielli E, De Amicis R, Barile A, Masciocchi C, Gallucci M. Spontaneous resolution of lumbar disk herniation: predictive signs for prognostic evaluation. Neuroradiology 2004;46:916-22.

17. Erginousakis D, Filippiadis DK, Malagari A, Kostakos A, Brountzos E, Kelekis NL, et al. Comparative prospective randomized study comparing conservative treatment and percutaneous disk decompression for treatment of intervertebral disk herniation. Radiology 2011;260:487-93.

18. Bokov A, Skorodumov A, Isrelov A, Stupak Y, Kukarin A. Differential treatment of nerve root compression pain caused by lumbar disc herniation applying nucleoplasty. Pain Physician 2010;13:469-80.

19. Woolf CJ, Doubell TP. The pathophysiology of chronic pain: increased sensitivity to low threshold $A \beta$-fibre inputs. Curr Opin Neurobiol 1994;4:525-34.

20. Jonsson B, Stromqvist B. Significance of a persistent positive straight leg raising test after lumbar disc surgery. J Neurosurg 1999;91(1 Suppl):50-3.

21. Knop-Jergas BM, Zucherman JF, Hsu KY, DeLong B. Anatomic position of a herniated nucleus pulposus predicts the outcome of lumbar discectomy. J Spinal Disord 1996;9:246-50.

22. Kim SY, Lee IS, Kim BR, Lim JH, Lee J, Koh SE, et al. Magnetic resonance findings of acute severe lower back pain. Ann Rehabil Med 2012;36:47-54.

23. Kang CH, Kim YH, Lee SH, Derby R, Kim JH, Chung $\mathrm{KB}$, et al. Can magnetic resonance imaging accurately predict concordant pain provocation during provocative disc injection? Skeletal Radiol 2009;38:877-85. 